\title{
Study of nuclear physics input-parameters via high-resolution $\gamma$-ray spectroscopy
}

\author{
Philipp Scholz ${ }^{1, \star}$, Felix Heim ${ }^{1}$, Jan Mayer ${ }^{1}$, Mark Spieker ${ }^{1}$, and Andreas Zilges ${ }^{1}$ \\ ${ }^{1}$ Institut für Kernphysik, Universität zu Köln, Zülpicher Str. 77, 50937 Köln, Germany
}

\begin{abstract}
For nucleosynthesis networks of isotopes heavier than those in the iron-peak region, reaction rates are often calculated within the Hauser-Feshbach statistical model. The accuracy of the predicted cross sections strongly depends on the uncertainties of the nuclear-physics input-parameters. These are nuclear-level densities, $\gamma$-ray strength functions, and particle+nucleus optical-model potentials. Here we report on the investigation of statistical properties of nuclei via radiative proton-capture reactions using highresolution $\gamma$-ray spectroscopy.
\end{abstract}

\section{Introduction}

The Hauser-Feshbach (HF) statistical model is widely used to predict reactions rates for nucleosynthesis process like the $r$ process [1] or the various $p$ processes [2]. Uncertainties in the predictions of these reaction rates can vary by orders of magnitude depending on the nuclear-physics models which are entering the HF calculations. Therefore, one key element in understanding the nucleosynthesis in stellar environments is a robust basis of models for nuclear-physics input as nuclear-level densities (NLDs) and $\gamma$-ray strength functions ( $\gamma \mathrm{SFs})[3]$.

In addition to their direct impact to reaction-network calculations, cross-section measurements of radiative charged-particle reactions can be a useful tool to investigate the statistical properties of nuclei. Using the high-efficiency $\gamma$-ray spectrometer HORUS at the Institute for Nuclear Physics at the University of Cologne [4], the $\gamma$-decay behavior of reaction products can be investigated with very high resolution. Here we report on two methods which can be used to study $\gamma$ SFs as well as NLDs; the measurement of partial (p, $\gamma$ ) cross sections and the study of two-step $\gamma$-ray cascades.

\section{Radiative proton-capture on ${ }^{92} \mathrm{Mo}$ and two step $\gamma$-ray cascades}

The isotope ${ }^{92} \mathrm{Mo}$ is the isotopically most abundant $p$ nucleus [5] and, therefore, attracted a lot of attention to its production sites and mechanisms [2]. As the inverse reaction of the $\gamma$-induced proton emission on ${ }^{93} \mathrm{Tc}$ within the $\gamma$ process, the ${ }^{92} \mathrm{Mo}(\mathrm{p}, \gamma)$ reaction is one of the most studied (see Ref. [6] and references therein). However, previous experiments using different techniques solely focused on low proton energies close to or inside the astrophysically relevant energy region. At these low energies huge fluctuations in the measured cross-section values were reported which hampered the comparison

^e-mail: pscholz@ikp.uni-koeln.de 
Figure 1. The extracted TSC matrix for ${ }^{92} \mathrm{Mo}$ for a proton energy of $5.1 \mathrm{MeV}$. Vertical cuts in the TSC matrix correspond to TSC spectra for the deexcitation to different levels in ${ }^{93} \mathrm{Tc}$ via two step $\gamma$-ray cascades. Two step cascade energies for the ground-state and the first two exited states are indicated by arrows.

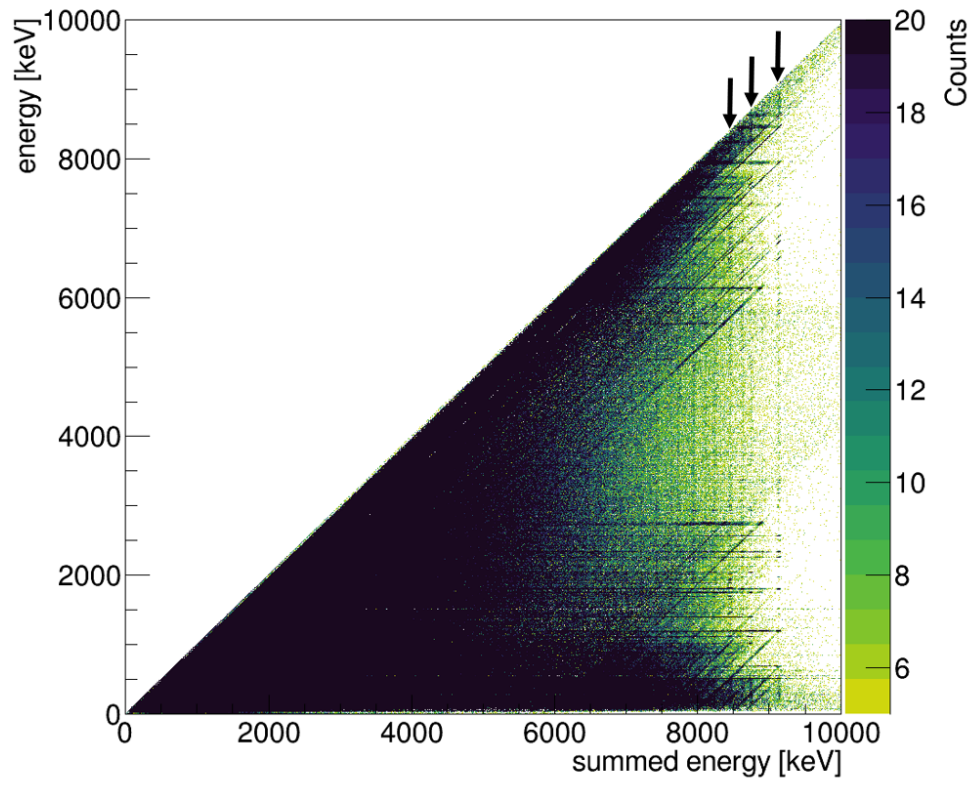

to HF calculations and, thus, conclusion on nuclear-physics models. Therefore, cross-section measurements were performed at proton energies between $3.7 \mathrm{MeV}$ and 5.3 MeV via the in-beam method at the HORUS $\gamma$-ray spectrometer [7]. The in-beam method with high-purity germanium detectors (HPGe) enables the determination of absolute total cross sections via the detections of all groundstate transitions in the reaction product. Additionally partial cross-sections for the radiative capture to excited levels can be determined (see, e.g, $[8,9]$ and references therein).

As it was shown for the ${ }^{89} \mathrm{Y}(\mathrm{p}, \gamma){ }^{90} \mathrm{Zr}$ reaction [10], partial cross-sections for the population of excited levels in the reaction product yield insight into the $\gamma$-strength of a nucleus. Different models for the $\gamma \mathrm{SF}$ combined with a specific NLD model and a proton+nucleus optical model potential can describe total reaction cross-sections well enough although they might not be able to describe the statistical $\gamma$-decays during the deexcitation of the compound nucleus. In contrast to that, partial crosssections yield information on the probability of $\gamma$-ray emission depending on the $\gamma$-ray energies.

For the case of ${ }^{92} \mathrm{Mo}(\mathrm{p}, \gamma)$ it was shown that a combination of M1-strength from shell model calculations and E1-strength from the Skyrme-/Gogny-HFB+QRPA model used for the HF calculations describe the total cross section much better than the default settings of the TALYS code (Standard Lorentzian/Constant Temperature model) [7]. However, none of the models were able to successfully describe all partial cross sections. Please note, that the comparison of HF calculation results to partialand total cross sections of radiative proton-capture reactions is not suitable for a direct determination of neither $\gamma$ SF nor NLD and, hence, other methods need to be used to confirm the results.

Another approach to verify $\gamma \mathrm{SF}$ and NLD models simultanously is the method of two step cascades (TSC) [11]. Originally developed for radiative neutron-capture reactions, this technique was applied recently to radiative proton-capture reactions as well [12] and served as additional confirmation for results obtained via the Oslo Method [13]. The advantage in the use of protons instead of neutrons is the smaller influence of Porter-Thomas fluctuations on the measured spectra [14]. 

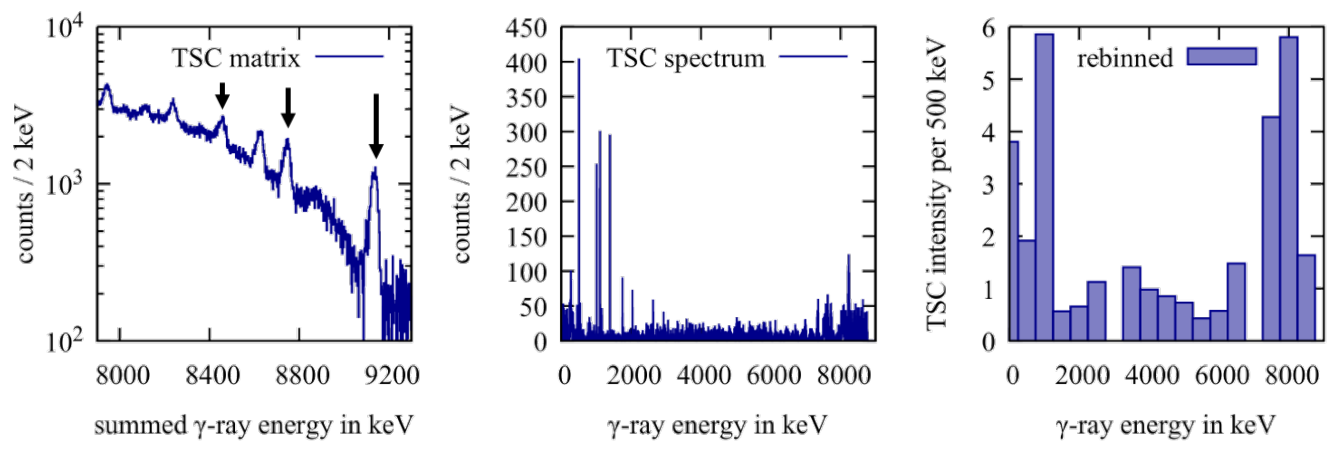

Figure 2. In the projection of the TSC matrix on to the axis of summed $\gamma$-ray energies (a), peaks for the population of different states via two-setp cascades in ${ }^{93} \mathrm{Tc}$ are visible. Via gating on of the summing peaks in the TSC matrix, the TSC spectrum (b) can be obtained showing the energies of the $\gamma$-rays as a part of the cascade. For the comparison to the results of modeling the TSC spectra based on different inputs for the $\gamma$-strength and leveldensity, TSC spectra are usually presented in wider energy bins, here $500 \mathrm{keV}$ (c).

The idea of this technique is the investigation of statistical $\gamma$-decay behavior via the detection of coincident $\gamma$-rays. If a spectrum of individual $\gamma$-rays of a two step cascade from an inital state $i$ to a final state $f$ via an intermediate state $m$ in the reaction product is measured, the intensity $I_{i f}\left(E_{1}, E_{2}\right)$ in the so-called TSC spectrum must follow the equation

$$
I_{i f}\left(E_{1}, E_{2}\right)=\sum_{X L, X L^{\prime}, J_{m}^{\pi}} \frac{\Gamma_{i m}^{X L}\left(E_{1}\right)}{\Gamma_{i}} \rho\left(E_{m}, J_{m}^{\pi}\right) \frac{\Gamma_{m f}^{X L^{\prime}}\left(E_{2}\right)}{\Gamma_{m}}+\sum_{X L, X L^{\prime}, J_{m^{\prime}}^{\pi}} \frac{\Gamma_{i m^{\prime}}^{X L}\left(E_{1}\right)}{\Gamma_{i}} \rho\left(E_{m^{\prime}}, J_{m^{\prime}}^{\pi}\right) \frac{\Gamma_{m^{\prime} f}^{X L^{\prime}}\left(E_{2}\right)}{\Gamma_{m^{\prime}}}
$$

where $\Gamma_{i m}^{X L}(E)$ is the $\gamma$-decay width for a $\gamma$-transition of character and multipolarity $X L$ and energy $E$ and $\rho(E, J)$ is the level density for a specific spin $J$ and parity $\pi$ at the intermediate energy $E_{m}$ [14]. Because the order of both $\gamma$-rays is indistinguishable, the term in Eq. 1 appears twice.

Since the digital data acquisition at the HORUS $\gamma$-ray spectrometer allows for the measurement of $\gamma \gamma$ coincidence data [4] the method can naturally be applied to data measured during previous experiments aiming for the determination of cross-section values for, e.g., ${ }^{92} \mathrm{Mo}(\mathrm{p}, \gamma)$.

At first, the so-called TSC matrix is extracted from the symmetric $\gamma \gamma$ coincidence matrix by sorting events with multiple hits in pairs into a 2D-histogram depending on the single and the total (summed) $\gamma$-ray energy (c.f. Fig. 1). As it can be seen in Fig. 1, the TSC matrix shows rather weak vertical lines amongst others at summed energies of $9.1 \mathrm{MeV}, 8.7 \mathrm{MeV}$, and $8.4 \mathrm{MeV}$ representing $\gamma$-ray cascades populating the ground-state, the first, and the second excited state in ${ }^{93} \mathrm{Tc}$, respectively.

Figure 2 shows from the left to the right an example of how the final TSC spectra can be obtained from the $5.1 \mathrm{MeV}$ measurement of the ${ }^{92} \mathrm{Mo}(\mathrm{p}, \gamma)$ reaction. In Fig. 2 a) the projection of the TSC matrix onto the summed energies is plotted showing summing-peaks for the population of different levels in ${ }^{93} \mathrm{Tc}$. Via gating on one of these summing-peaks, the spectrum of two step $\gamma$-ray cascades populating the specific level can be obtained (c.f. Fig. 2 b)). In the low energy and high-energy part of these TSC spectra discrete $\gamma$-ray transistion from excited states in ${ }^{93} \mathrm{Tc}$ to the ground state and the prompt $\gamma$-rays from the entry state to a intermediate state are visible, respectively. At medium energies, statistical $\gamma$-ray transitions appear whose intensities strongly depend on the $\gamma$ SF and NLD of ${ }^{93} \mathrm{Tc}$. Once the TSC spectrum is corrected for coincidence-efficiencies, normalized onto the absolute number of proton captures, and rebinned, the symmetric nature of the TSC spectrum becomes apparent 
(see Fig. 2 c)) and the TSC intensity of the energy bins can be compared to Eq. 1. Usually this is done utilizing the DICEBOX algorithm [15] by varying the input for $\gamma$ SF and NLD. Via such comparisons structures like the low-energy upbend [12] or scissor modes [16] in the $\gamma \mathrm{SF}$ have already been investigated.

\section{Measurement of ${ }^{63} \mathrm{Cu}(p, \gamma \gamma){ }^{64} \mathrm{Zn}$ and ${ }^{65} \mathrm{Cu}(p, \gamma \gamma){ }^{66} \mathrm{Zn}$}

Recently, the first radiative proton-capture experiment at the HORUS $\gamma$-ray spectrometer was specifically performed for applying the TSC method to the ${ }^{63} \mathrm{Cu}(\mathrm{p}, \gamma \gamma)^{64} \mathrm{Zn}$ and ${ }^{65} \mathrm{Cu}(\mathrm{p}, \gamma \gamma){ }^{66} \mathrm{Zn}$ reactions at 3.5 MeV and 2.0 MeV, respectively. The beam energies were chosen to be below the respective (p,n)reaction thresholds to suppress any contribution from this channel. With Q-values of 7.7 MeV and 8.9 MeV, NLD and $\gamma \mathrm{SF}$ models can be studied up to excitation energies of $11.2 \mathrm{MeV}$ and $10.9 \mathrm{MeV}$, respectively.

In both cases, summing-peaks for the populations of several excited states in the reaction products were observed and can be used to extract TSC spectra. Until the the final TSC intensities can be compared to theory, a careful investigation of the background in the TSC spectra has to be carried out via GEANT4 simulations. However, the application of the TSC method to the data obtained with the HORUS $\gamma$-ray spectrometer seems promising and final results for the studied reactions should be available soon.

\section{Acknowledgment}

The authors thank A. Dewald and the accelerator staff at the Institute for Nuclear Physics in Cologne for providing excellent beams. This project was supported by the Deutsche Forschungsgemeinschaft under Contract No. ZI 510/8-1 and the ULDETIS project within the University of Cologne Excellence Initiative institutional strategy. J.M. and P.S. are supported by the Bonn-Cologne Graduate School of Physics and Astronomy.

\section{References}

[1] M. Arnould, S. Goriely, and K. Takahashi, Phys. Rep. 450 (2007) 97.

[2] M. Arnould and S. Goriely, Phys. Rep. 384 (2003) 1.

[3] T. Rauscher, Int. J. Mod. Phys. E 20 (2011) 1071.

[4] L. Netterdon et al., Nucl. Instr. Meth. A 754 (2014)94.

[5] Michael Berglund and Michael E. Wieser, Pure Appl. Chem. 83 (2011) 397.

[6] G. Gyürky et al. Nucl. Phys. A 922 (2014) 112.

[7] J. Mayer et al., Phys. Rev. C 93 (2016) 045809.

[8] A. Sauerwein et al., Phys. Rev. C 86 (2012) 035802.

[9] S. Harissopulos. et al., Phys. Rev C 93 (2016) 025804.

[10] L. Netterdon et al., Phys. Lett. B 744 (2015) 358.

[11] F. Bečvár̆ et al., Phys. Rev. C 46 (1992) 1276.

[12] A. Voinov et al., Phys. Rev. Lett. 93 (2004) 142504.

[13] A. Schiller, L. Bergholt, M. Guttormsen, E. Melby, J. Rekstad, and S. Siem, Nucl. Instrum. Methods Phys. Res. A 447, 498 (2000).

[14] A. Voinov et al. EPJ Web of Conferences 93, 01022 (2015).

[15] F. Bečvár, Nucl. Instr. Meth. A 417, 434 (1998).

[16] F. Bečvár̆ et al., Nucl. Instr. Meth. B 261 (2007) 930. 